\title{
A Novel Approach to Road Mapping
}

\section{Ishu Verma ${ }^{1}$ and Kuldeep Singh ${ }^{2}$}

\begin{abstract}
The correct and timely update of road map databases is important in many Geographic Information System (GIS) applications for navigation information (distance, speed, direction etc.), traffic management, urban planning, road monitoring etc. Road map detection is one of the great application in which researchers are showing interest to detect the new built road and update database to help people. In this paper we have presented a road map detection technique using snakes counter model which is based on junction point. As we know that large number of vehicles positioning systems are already available in market and most of them are GPS based systems. But the reliability is still in problem. In urban areas GPS is difficult to use due to satellite signal blockage and multipath effects caused by surrounding buildings. In this paper we will introduce new technique based on intersection points of road called as juction point baesd on snakes counter model. This technique is used to improve the integrity and reliability of map matching technique.
\end{abstract}

Keywords: road map, map matching, junction points

\section{Introduction:}

Roadmap displays map of streets, new built roads and tracks the position provided by GPS receiver. With the increasing technology, its excellence has increased considerably in the last decades. It is an alternate to expensive road survey. This is a method to infer the road network from GPS data logged from regular vehicles.First step is to find the road intersection and non intersection points. Then we will describe how these points on the road map are located.

As we know that by using one type of road feature it is hard to get excellent extraction effects. In order to get good results multiple methods are used according to the real time application. Roads are the backbone and essential modes of transportation and it provides many different supports for human civilization. At present, most of the road maps are produced and updated by some companies fielding convoys of some specialized vehicles that are outfitted with GPS (Global Positioning System) to drive the roads and record data but this is a very costly method and not easy to update with changes in the road network [3]. 
In the geometrics' field, there are many alternatives to discover and update a road network, which includes ground survey, comparing the vector map, image processing based updating, etc. Image processing updating is done mostly via feature extraction from the obtained satellite image, due to its various properties such as high spatial resolution, fast orbit repeatability, rich multi-spectrum information etc., this method has gained popularity [2].

Road map detection and updating includes the following procedures.

Feature Extraction: If the input data is too large and contains some redundant data, then it is transformed into a reduced features set called Feature vector. Only those features are selected which contains the relevant information and others are removed. For example, in road map detection we need only roads and the other information like buildings, parks etc are removed. This can be done by using some filters. In this papermedian filters are used for the purpose [4].

The difficulties of road extraction lies in the image characteristics of road features can be affected by sensor type, spectral and spatial resolution, weather, light variation and ground characteristics.

Morphological image processing: Morphological image processing is a collection of various non linear operations related to the morphology of features in image. These operations depend only on the relative ordering of pixels and not on their numerical values. So these are best suited for binary images [5].

Image thinning: Image thinning is a morphological operation which is performed to remove the selected foreground pixels from a binary image, It is used to clean the output of edge detectors by reducing lines to a single pixel [6].we have make image enhancement to extract useful information from the image. At last the result of matching describes whether update is required or not.

\section{Previous work:}

M. Butenuth et al. [7] has studied the extraction of roads from the high resolution spatial images of suburban scenes by applying segmentation on images using the Normalized Cuts algorithm. The segmentation is done in order to yield a fine division between road area and its surroundings. The Normalized Cuts algorithm, is a graph-based technique which divides the image on the basis of pixel similarities. The definition of these similarities can include several features, which is vital for the segmentation in images having complex surroundings such as buildings, parks etc. The segmentation is done on the bases of color, hue, edges and road color derived with prior information about the location of the centerline from the database.

M.Rajeswari et al. [8] has proposed two approaches for road network extraction for an urban area. If an Image is considered in original form as it is received, then it becomes difficult and computationally costly to extract roads due to presence of other road-like features having straight edges. Hence roads are first extracted as elongated regions by removing bright regions (that mostly represent the buildings, parking lots, other open spaces), after this non-linear noise segments are removed by using median filtering based on the fact that the road networks contain huge number of small linear structures.

O.Sharma et al. [9] has presented an approach in attempt to automate the process of extracting feature boundaries from satellite imagery. The given approach is intended to replace manual digitization by a 
computer assisted boundary detection, and conversion to a vector layer in a GIS. Although a lot of work is already done in this direction, but his research work is different from the previous ones in the way that it uses the Delaunay graph and the Verona tessellation for boundary extraction and skeletons that are guaranteed to be topologically equivalent to the segmented objects.

Volodymyr Mnih and Geoffrey E. Hinton [10] proposed a method to detect roads using neural networks with millions of trainable weights which appears at a much larger context than was used in previous attempts at learning the task. The neural network is trained on a large amount of data using a consumer GPU. They have demonstrated that the predictive performance can be substantially increased by initializing the feature detectors using recently developed unsupervised learning methods also by taking an advantage of the local spatial coherence of the output labels. They have demonstrated that the proposed method works reliably on two challenging urban datasets that are an order of magnitude larger than what was used to evaluate previous techniques.

\section{Proposed work:}

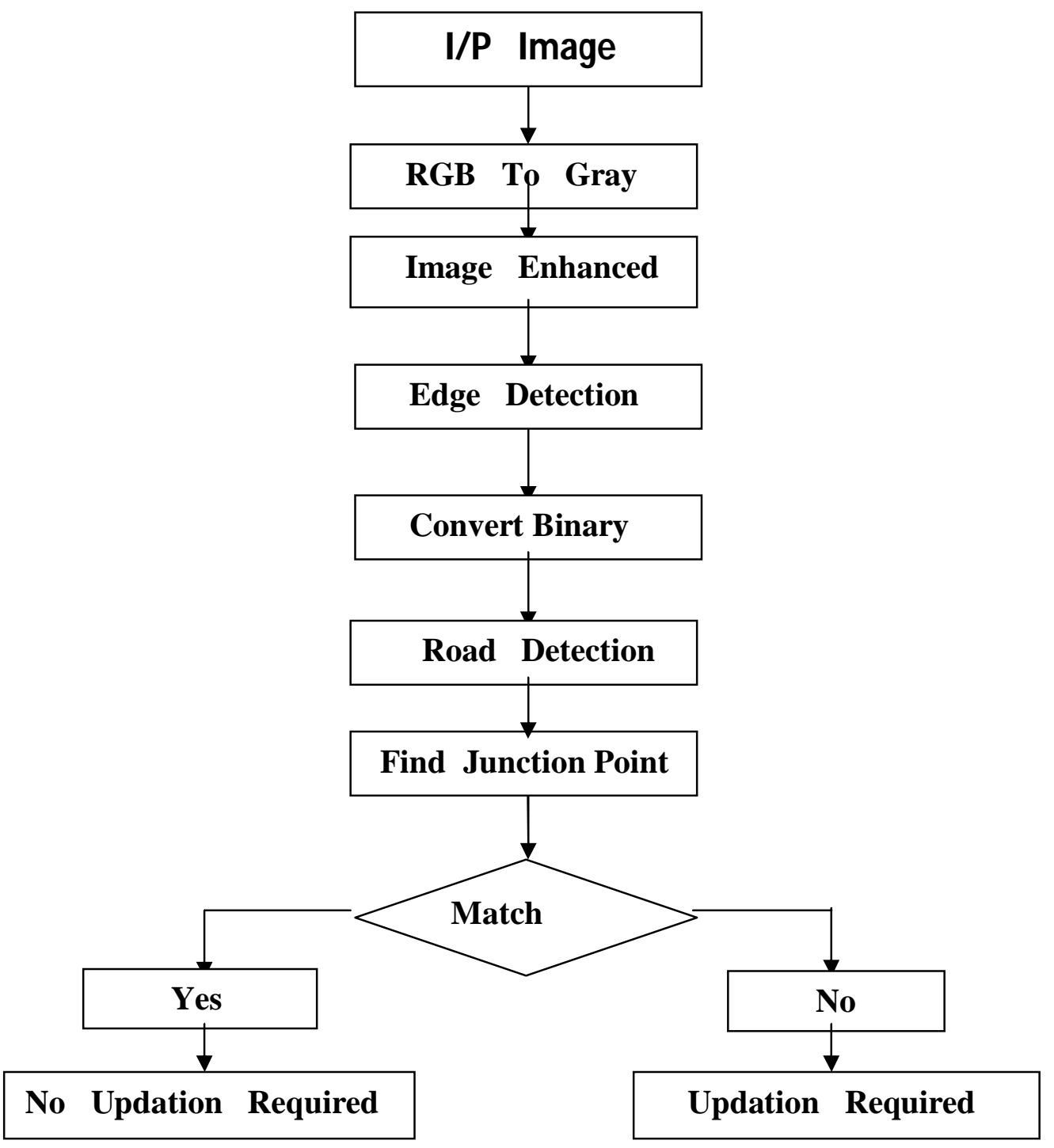

Fig1. Work flow diagram for proposed method 
Input Image:first work is to upload an image to the system. This image can be in any format e.g. JPG, PNG, BMP. This image can be taken either from previous database or current video or manually. The imagesare uploaded to compare with the database. This image is called as test image.

RGB to Gray: in second step test image is converted to the grayscale using Luminosity method By eliminating the hue and saturation information while retaining the luminance. If we have parallel computing toolbox installed then RGB to gray can perform this conversion on GPU. (i)

Image Enhancement:this step is followed if the image is not clear or blurred. In this case we need to enhance our image and if enhancement in image is required then we will use median filter for image enhancement. It is used to extract useful information from road network.

Convert to binary: Grayscale image is converted to binary using threshold rule. To use this rule we put a threshold value for each pixel and if the value is greater than this threshold value then this will be done as 1 . And if the value is smaller than threshold value then it will be taken as 0 .

Road Detection:Road is expressed or represented with the help of white intensity points and other parts are left black. To ignore the greenery beside the road they are converted to the black intensity points and the road in between the white lines is coloured white. In this we have used autointial snake model by fixing its initial point as corner points. This method is combined with the junction point method to identify the intersection of roads.

Find Junction Point: Junctions points are calculated to find the intersection of the road.

Matching of Image: Matching is decided by calculating the white and black intensity points of the image. The test image is matched with the training images present in the database already. This is compared depending upon the matching percentage the road map is to be update or not.

The figure below (fig 2) shows the work done in the proposed technique.

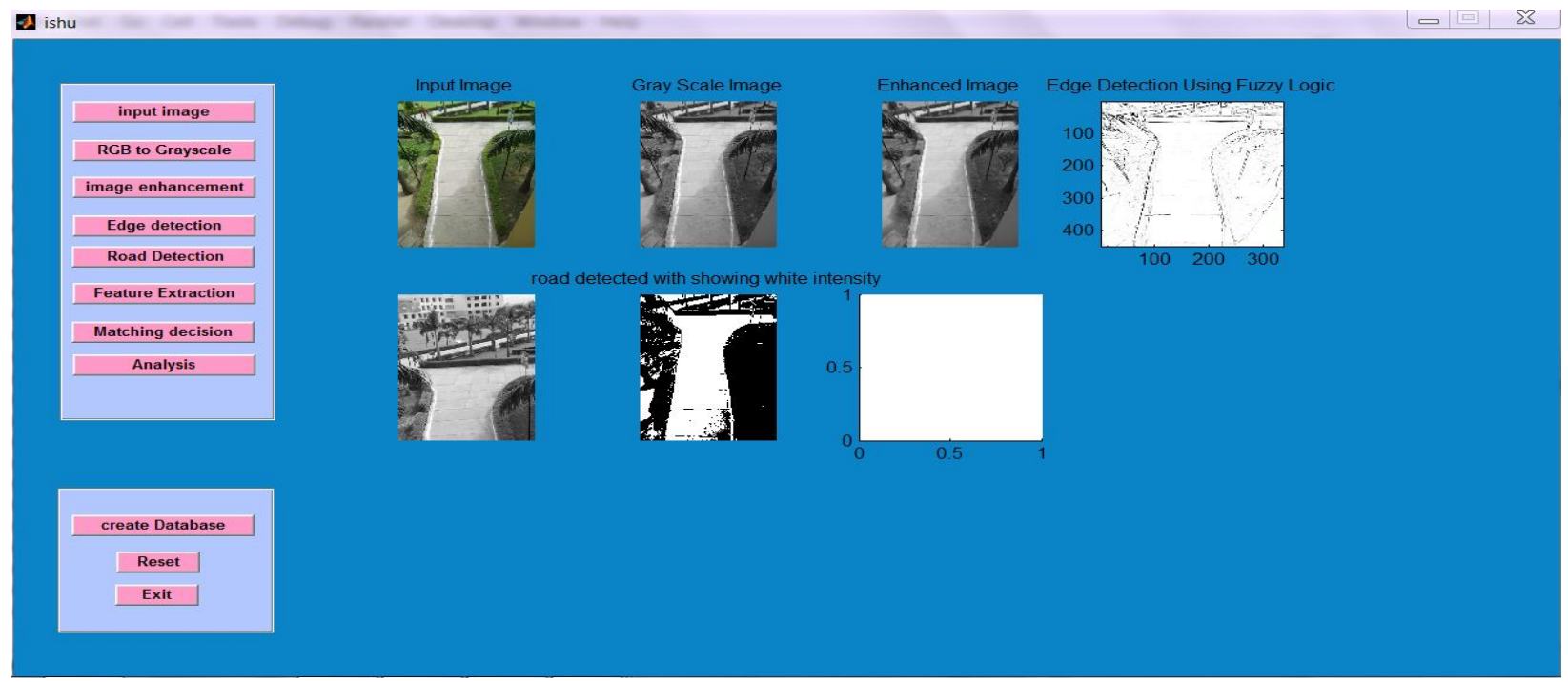

Fig2. The work flow of proposed work 


\section{Results}

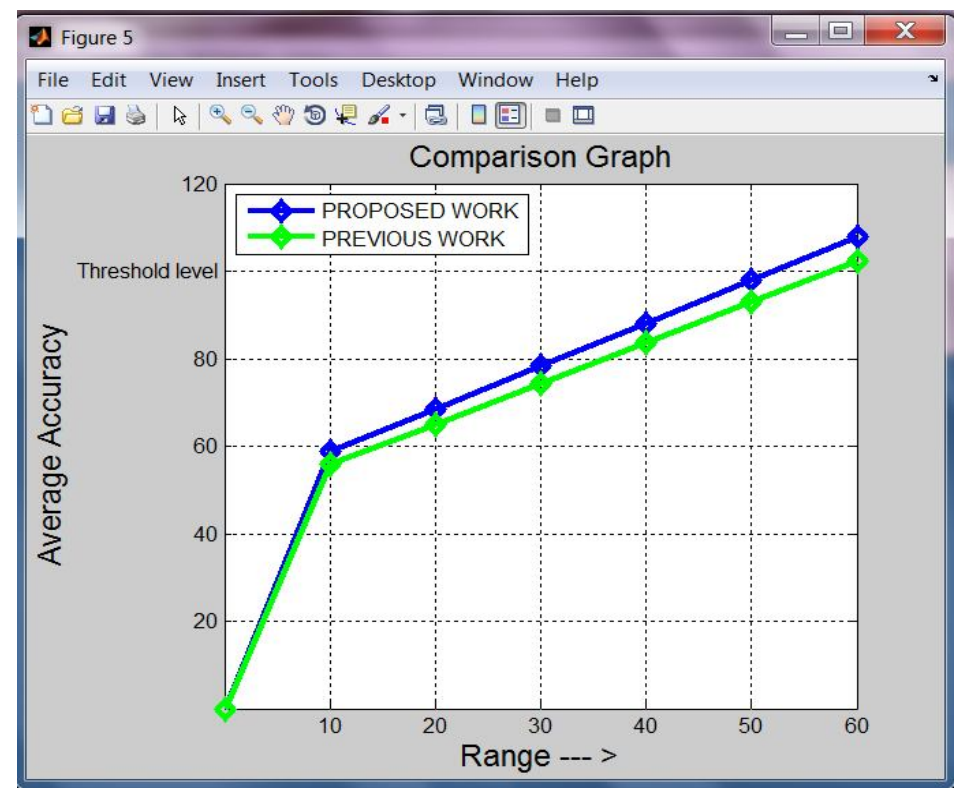

Fig 3. Comparison between the Accuracy of proposed work and the previous work

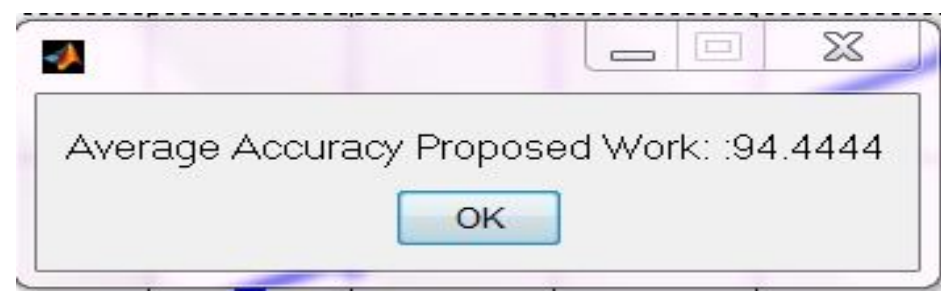

Fig. 4 Average accuracy of the proposed work

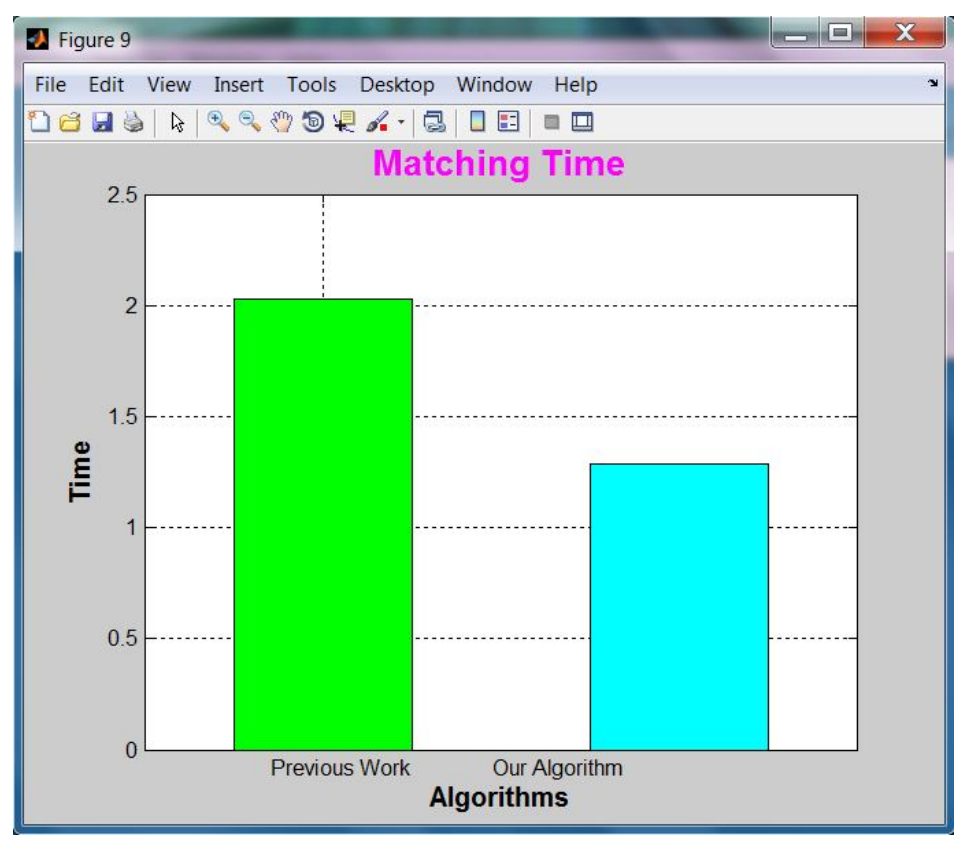

Fig 5. Comparison between the matching of given approach and previous approach 
Figure 3 and 4 gives the comparison between the proposed work and the previous work, in terms of accuracy and matching time. The main concern in this paper is about optimizing the accuracy of the system. The results show that the accuracy of the proposed work is higher than the previous algorithm. So, the given method seems to be a promising technique for road map detection. Moreover, with the increased the accuracy of the work, the matching time is also decreased as compare to the previous work. The accuracy and matching time are $94.4 \%$ (Fig. 4) and $1.28 \mathrm{~s}$ (Fig. 5) respectively.

\section{References:}

[1] F. Heinzle, K.-H. Anders, M. Sester, "AUTOMATIC DETECTION OF PATTERNS IN ROAD NETWORKS-METHODS AND EVALUATION", Institute of Cartography and Geoinformatics, University of Hannover

[2] Hu, X.,Tao, C.V., "Automatic Extraction of Main-Road from High Resolution Satellite Imagery", IAPRS, VOLUME XXXIV, PART 2, COMMISSION II, Xi'an, Aug.20-23,2002

[3] Alireza Fathi , John Krumm, "Detecting Road Intersections from GPS Traces", GIScience 2010, Sixth International Conference on Geographic Information Science, Zurich, 14-17th September, 2010

[4] https://en.m.wikipedia.org/wiki/Feature_extraction

[5] https://www.cs.aukland.ac.nz/courses/compsci773s1c/lectures/Imageprocessing-html/topic4.htm

[6] http://homepages.inf.edu.ac.uk/rbf/HIPR2/thin.htm

[7] A.Grote, M.Butenuth, C.Heipke , "Road extraction in suburban area based on normalized cuts", International archives of photogrammetry, remote sensing and spatial information sciences, pp. 51-56, 2007.

[8] M.Rajeswari, K.S.Gurumurthy, S.N.Omkar, L.Pratap Reddy, "Automatic Extraction of Road Networks based on Normalized cuts and Mean shift method for high resolution satellite imagery", International journal of computer applications, Vol.18-No.7, pp. 10-16, 2011.

[9] O.Sharma, D.Mioc, F.Anton ,"Polygon feature extraction from satellite imagery based on colour image segmentation and medial axis", The international archives of the photogrammetry, remote sensing and spatial information sciences, Vol.38, pp. 235-240, 2008.

[10] Volodymyr Mnih and Geoffrey E. Hinton, "Learning to Detect Roads in High-Resolution Aerial Images", Department of Computer Science, University of Toronto 